\title{
Stiff Person Syndrome
}

National Cancer Institute

\section{Source}

National Cancer Institute. Stiff Person Syndrome. NCI Thesaurus. Code C85170.

A rare neurologic disorder characterized by muscle rigidity. It usually affects the axial

muscles and eventually extends to the proximal limb muscles. It is associated with muscle spasms which may lead to muscle damage and fractures. 